\title{
Whole body vibration improves body mass, flexibility and strength in previously sedentary adults
}

\author{
Aayesha Kholvadia, Maryna L Baard \\ Department of Human Movement Science, Nelson Mandela Metropolitan University, Port Elizabeth \\ Aayesha Kholvadia, MA Human Movement Science (Biokinetics) \\ Maryna L Baard, DPhil
}

Corresponding author: M L Baard (maryna.baard@nmmu.ac.za).

\begin{abstract}
Objectives. This study aimed to determine the effectiveness of whole body vibration (WBV) training for promoting healthrelated physical fitness in sedentary adults.

Design. A non-randomised sampling technique was used with an equivalent match-pair comparison group, pre- and posttest design. Volunteers were gathered through a combination of accidental and snowball sampling and divided into either the experimental $(n=32)$ or control group $(n=30)$. Dependent variables included body mass, hamstring flexibility as measured by the sit-and-reach test, upper-body strength as measured by a grip strength dynamometer, abdominal and upper-body muscular endurance as measured by 1-minute timed sit-up and push-up tests, respectively. The standardised YMCA fitness battery was used as the evaluation protocol. The WBV experimental group participated in a progressive 3 times/week training programme for a maximum duration of 30 minutes/session for 12 consecutive weeks. The control group remained sedentary.

Results. Significant improvements in all five of the selected dependent parameters were measured.

Conclusion. WBV training 3 times weekly for 30 minutes/ session provides an effective method of exercise intervention for health promotion in sedentary adults over a 12 -week period.
\end{abstract}

S Afr J SM 2012;24(2):60-64.

\section{Introduction}

In South Africa physical inactivity presents a challenge that must be addressed urgently since an excessively high proportion (50\%) of South Africans are currently living sedentary lives. ${ }^{1}$ Inactivity in adults increases the risk of non-communicable diseases, and because these are chronic diseases of lifestyle they negatively affect quality of life. ${ }^{2,3}$ For sedentary individuals, participation in moderate-intensity physical activity is advisable because such activity would reduce the risk of coronary heart disease (CHD) and have substantial healthrelated fitness benefits; not only those associated with obesity, but also the reduction of blood pressure, improved levels of high-density lipoproteins and the control of blood glucose in overweight people, even without significant weight loss, and a reduction in the risk of colon cancer and, among women, breast cancer. ${ }^{3,4}$

Therefore sedentary adults need to be persuaded to exercise regularly at a moderate intensity. ${ }^{2,5}$ The challenge however is to overcome barriers related to exercise participation, including lack of compliance, poor weather conditions, time constraints, work obligations, and low levels of motivation, confidence and self-esteem. ${ }^{6}$

The use of whole body vibration (WBV) as an exercise intervention for health promotion among sedentary adults is increasingly being considered among health professionals because, compared with conventional training methods, it offers a way of training with practical, physical and psychological advantages. WBV is a current neuromuscular training method, which even at a low intensity provokes muscle length changes that stimulate the sensory receptor of the muscle spindle, thereby eliciting a tonic vibratory reflex, which is defined as a sustained contraction of a muscle subjected to vibration. WBV intervention exploits the body's innate reflex response to disruptions in stability in order to stimulate and enhance muscle strength and performance. Practically, WBV training has the advantage of overcoming some of the cited obstacles to exercise because it decreases overall training time and takes place indoors. More importantly, it offers a host of physical benefits such as improved muscular strength, flexibility, range of motion, bone density, and improved blood circulation. ${ }^{7}$ In addition, WBV heightens confidence, which encourages ongoing participation and programme adherence., ${ }^{2,8}$

Given these advantages, a WBV exercise programme, if individually structured and supervised, provides an attractive mode of training for sedentary individuals. The purpose of this investigation is to determine the effectiveness of WBV training in enhancing the following components of health-related physical fitness in sedentary individuals: body mass, hamstring flexibility, upper-body strength and abdominal and upper-body muscular endurance.

\section{Methods}

A quasi-experimental approach using a two-group comparison, preand post-test design, was utilised to compare the responses of the experimental and control group over a 12 -week intervention period for selected health-related physical fitness parameters of measures of body mass, hamstring flexibility, grip strength, and abdominal and upper-body muscle endurance. ${ }^{7}$ A non-randomised sampling technique ${ }^{8}$ was used with an equivalent match-pair design to divide the base of volunteers gathered through a combination of accidental and snowball sampling into either the experimental $(n=32)$ or control group $(n=30)$. The match-pair design was used to ensure that the dynamics within the experimental and control groups were uniform prior to the WBV intervention. In order to match individuals, each participant received a composite score after completing the healthrelated physical fitness evaluation at the pre-test. Participants with the same or similar composite scores were then allocated to either the experimental or control group. Each group therefore resembled 
the other in as many parameters as possible. ${ }^{9,10}$ The Nelson Mandela Metropolitan University (NMMU) Research Human Ethical Committee gave ethical approval. The exercise intervention occurred 3 times a week for 12 consecutive weeks.

\section{Data-gathering techniques}

Prospective participants were informed of the study by electronic mail, highlighting the rationale of the study and specifying the inclusion criteria as being sedentary for a period of 6 months. Exclusion criteria were clearly defined because of the possible contraindications outlined by the Power Plate ${ }^{\oplus}$ manufacturers. All NMMU staff were invited to participate in the study on a voluntary basis. Table 1 indicates descriptive data about the participants. Prior to measurement of the dependent variables, a health risk assessment was done by screening for CHD risk factors as measured by the Physical Activity Readiness Questionnaire (PAR-Q). This was done to ensure safe participation in the 12week intervention programme. The dependent variables included body mass, hamstring flexibility as measured by the sit-andreach test, upper-body muscle strength as measured by a grip strength dynamometer, and abdominal and upper-body muscle endurance as measured by 1 -minute timed sit-up and push-up tests, respectively. The well-known standardised YMCA fitness battery $^{2}$ was used as the evaluation protocol for both the pre-test and post-test assessments for both groups.

Table 1. Description of participants

\begin{tabular}{|c|c|c|}
\hline & $\begin{array}{l}\text { Experimental } \\
\text { group }\end{array}$ & Control group \\
\hline $\begin{array}{l}\text { Number of participants at the } \\
\text { beginning of the study, } n\end{array}$ & 42 & 30 \\
\hline Age of participants (range), years & $19-52$ & $17-54$ \\
\hline Gender delineation of participants & $\begin{array}{l}21 \text { males, } \\
21 \text { females }\end{array}$ & $\begin{array}{l}15 \text { males, } \\
15 \text { females }\end{array}$ \\
\hline $\begin{array}{l}\text { Number of participants at the end of } \\
\text { the study, } n\end{array}$ & 32 & 30 \\
\hline Dropout rate, $\%$ & 24 & 0 \\
\hline
\end{tabular}

The following procedure was employed: written consent was obtained from each participant prior to the study; clinical data were gathered; pre-test measurements were applied for the dependent variables; the WBV intervention programme was implemented (or a sedentary lifestyle was maintained for the control group) for a 12week period; the post-test measurement concluded the programme.

\section{Intervention programme}

WBV training, in the form of static exercises, was performed on the vibration platform (Power Plate) by the experimental group, while the control group remained sedentary throughout the 12 week intervention period. The experimental group trained with progressive increments in either the intensity (amplitude of the vibration) or duration resulting in an increase in the total length of the particular exercise session. Training intensity was manipulated by either increasing the amplitude of the vibration from low $(\mathrm{L})$ to high $(\mathrm{H})$, or by manipulating the intensity of the training between $30 \mathrm{~Hz}$ and $50 \mathrm{~Hz}$. Manipulation of either the total duration of the time spent on the vibration platform or the total duration of the exercise session was used to indicate the training progression. The training frequency was set at three training sessions per week. Aforementioned variables in the WBV intervention programme are displayed in Table 2. The programme design is based on the guidelines for training principles as prescribed by the American College of Sports Medicine (ACSM). ${ }^{11}$

\section{Statistical analysis}

The Statistica version 9.0 computer processing package (StatSoft, Inc, Tulsa, OK, USA) was used to analyse the data and the level of significance was set at $p<0.05$. For the comparisons involving the WBV experimental and sedentary control group, descriptive measures of means and standard deviations (SDs) were calculated. Independent $t$-tests and $\chi^{2}$ tests were performed to determine statistically significant intergroup differences at the pre- and post-tests for all selected dependent variables. Cohen's d-values were calculated to express the level of practical significance. The interpretation of Cohen's $d$-values is as follows: $d=0.20,0.50$ and 0.80 , respectively, indicate small, medium and large effects in practical significance. ${ }^{10,12}$

Table 2. WBV training variables

\begin{tabular}{|c|c|c|c|c|c|}
\hline \multicolumn{3}{|c|}{ Training intensity } & \multicolumn{2}{|r|}{ Training duration } & \multirow[t]{2}{*}{ Training type } \\
\hline Session (week) & Amplitude & $\mathrm{Hz}$ & Per exercise (s) & Total session duration (h:min:s) & \\
\hline 1 & $\mathrm{~L}$ & 30 & 30 & 0:00:10 & General: calf raise, lunge, abductor, adductor squeeze, squat \\
\hline 2 & $\mathrm{~L}$ & 30 & 45 & $0: 12: 30$ & \multirow{2}{*}{$\begin{array}{l}\text { Upper-body strength: push-up, bicep curl, side raise, tricep pull, } \\
\text { tricep dip }\end{array}$} \\
\hline 3 & $\mathrm{~L}$ & 30 & 60 & 0:21:00 & \\
\hline 4 & $\mathrm{H}$ & 30 & 45 & $0: 17: 15$ & \multirow{3}{*}{$\begin{array}{l}\text { Hamstring flexibility: hamstring stretch, quadriceps stretch, calf } \\
\text { stretch } \\
\text { Core: frontal bridge, side bridge, sit-ups }\end{array}$} \\
\hline 5 & $\mathrm{H}$ & 30 & 45 & $0: 17: 15$ & \\
\hline 6 & $\mathrm{H}$ & 35 & 45 & $0: 19: 30$ & \\
\hline 7 & $\mathrm{H}$ & 40 & 45 & 0:20:30 & \\
\hline 8 & $\mathrm{H}$ & 50 & 45 & $0: 20: 15$ & \\
\hline 9 & $\mathrm{H}$ & 30 & 60 & 0:30:00 & \\
\hline 10 & $\mathrm{H}$ & 35 & 60 & 0:30:00 & \\
\hline 11 & $\mathrm{H}$ & 40 & 60 & 0:30:00 & \\
\hline 12 & $\mathrm{H}$ & 50 & 60 & 0:30:00 & \\
\hline
\end{tabular}


Table 3. Pre- and post- test results based on mean differences of the selected five dependent variables for both groups

\begin{tabular}{|c|c|c|c|c|}
\hline Measurement & Group & Pre & Post & Post-pre difference \\
\hline \multirow[t]{5}{*}{ Body mass (kg) } & Control $(n=30)$ & $74.6 \pm 13.7$ & $74.9 \pm 13.8$ & $0.3 \pm 1.4$ \\
\hline & WBV $(n=32)$ & $74.5 \pm 19.0$ & $73.4 \pm 18.4$ & $-1.1 \pm 2.7$ \\
\hline & Difference & -0.1 & 1.5 & 1.4 \\
\hline & $t$-test; $p$-value & $-0.02 ; 0.985$ & $0.36 ; 0.719$ & $0.76 ; 0.507$ \\
\hline & Effect size & n.a. & n.a. & n.a. \\
\hline \multirow[t]{5}{*}{ Hamstring flexibility $(\mathrm{cm})$} & Control $(n=30)$ & $27.15 \pm 11.30$ & $26.29 \pm 11.16$ & $-0.87 \pm 1.84$ \\
\hline & WBV $(n=32)$ & $22.53 \pm 11.41$ & $27.32 \pm 7.86$ & $4.79 \pm 5.56$ \\
\hline & Difference & 4.60 & -1.10 & -5.66 \\
\hline & $t$-test; $p$-value & $1.59 ; 0.116$ & $-0.45 ; 0.652$ & $-5.31 ; 0.0001$ \\
\hline & Effect size & n.a. & n.a. & 1.35 large \\
\hline \multirow[t]{5}{*}{ Grip strength (kgf) } & Control $(n=30)$ & $47.8 \pm 25.2$ & $47.3 \pm 23.9$ & $-0.5 \pm 6.0$ \\
\hline & WBV $(n=32)$ & $41.0 \pm 18.8$ & $47.2 \pm 18.7$ & $6.2 \pm 5.2$ \\
\hline & Difference & 6.8 & 0.2 & -6.6 \\
\hline & $t$-test; $p$-value & $1.21 ; 0.229$ & $0.04 ; 0.971$ & $-4.67 ; 0.0001$ \\
\hline & Effect size & n.a. & n.a. & 1.19 large \\
\hline \multirow{5}{*}{$\begin{array}{l}\text { Abdominal muscle endurance } \\
\text { (reps) }\end{array}$} & Control $(n=30)$ & $25 \pm 13$ & $25 \pm 14$ & $0 \pm 2$ \\
\hline & WBV $(n=32)$ & $33 \pm 10$ & $37 \pm 9$ & $4 \pm 7$ \\
\hline & Difference & 8 & -12 & -5 \\
\hline & $t$-test; $p$-value & $0.24 ; 0.810$ & $-3.76 ; 0.000$ & $-4.12 ; 0.0001$ \\
\hline & Effect size & n.a. & 0.95 large & 1.05 large \\
\hline \multirow{5}{*}{$\begin{array}{l}\text { Upper body muscle endurance } \\
\text { (reps) }\end{array}$} & Control $(n=30)$ & $20 \pm 13$ & $19 \pm 12$ & $-1 \pm 2$ \\
\hline & WBV $(n=32)$ & $24 \pm 12$ & $33 \pm 14$ & $9 \pm 7$ \\
\hline & Difference & -4 & -14 & -10 \\
\hline & $t$-test; $p$-value & $-1.40 ; 0.166$ & $-3.90 ; 0.000$ & $-6.88 ; 0.000$ \\
\hline & Effect size & n.a. & 0.99 large & 1.75 large \\
\hline
\end{tabular}

\section{Results}

\section{Body mass}

As shown in Table 3, a decrease in body mass (kg) for the experimental group was measured, with a pre-test mean of $74.5 \mathrm{~kg}$ and a post-test mean of $73.4 \mathrm{~kg}$. The mean body mass for the control group ranged from $74.6 \mathrm{~kg}$ to a post-test value of $74.9 \mathrm{~kg}$. The experimental group showed a decrease of $1.1 \mathrm{~kg}$ in the mean body mass whereas the control group showed an increase of $0.3 \mathrm{~kg}$. No statistical significance was achieved as indicated by $t=0.10, p<0.05$ and $\chi^{2}=12.23, p<0.05$ for this component of body composition. Furthermore, moderate practical significance was indicated by Cohen's $d=0.60$. These results are reflected in Table 3.

A graphical representation of all five dependent variables based on the rank order of the mean pre- and post-test differences is displayed in Fig. 1.

\section{Hamstring flexibility}

As shown in Table 3, a significant increase in hamstring flexibility was measured for the experimental group, with a pre-test mean of $22.5 \mathrm{~cm}$ and a post-test mean of $27.3 \mathrm{~cm}$. Hamstring flexibility for the control group ranged between $27.1 \mathrm{~cm}$ and $26.2 \mathrm{~cm}$ for both the pre- and post-test analyses. The mean hamstring flexibility for the experimental group showed a statistically significant increase with $t=-5.31, p<0.05$ and $\chi^{2}=23.31, p<0.05$ after the 12-week intervention. Large practical significance was indicated by Cohen's $d=1.35$ as shown in Table 3 .

\section{Muscle strength Grip strength}

Table 3 reflects a significant increase in the grip strength values for the experimental group, with a pre-test mean of $41.0 \mathrm{kgf}$ and a post-test mean of $47.1 \mathrm{kgf}$. The experimental group showed a mean increase in grip strength by $6.1 \mathrm{kgf}$ whereas the control group showed a mean decrease of $0.4 \mathrm{kgf}$ having a pre-test mean value of $47.8 \mathrm{kgf}$ and a post-test value of $47.3 \mathrm{kgf}$. Grip strength for the experimental group showed a statistically significant increase with $t=4.67, p<0.05$ and $\chi^{2}=22.94, p<0.05$ after the 12week intervention. Large practical significance was indicated by Cohen's $d=1.19$ as indicated in Table 3 .

\section{Muscular endurance}

\section{Abdominal muscular endurance}

Table 3 reflects a significant increase in abdominal muscular endurance for the experimental group, with a pre-test mean of 33 repetitions and a post-test mean of 37 repetitions. Abdominal muscular endurance for the control group was 25 repetitions for both the pre- and post-tests. The mean muscular endurance values for the experimental group showed a statistically significant increase with $t=-$ 4.12, $p<0.05$ and $\chi^{2}=30.06, p<0.05$ after the 12-week duration of the study. Large practical significance was indicated by Cohen's $d=1.0$ as shown in Table 3. 


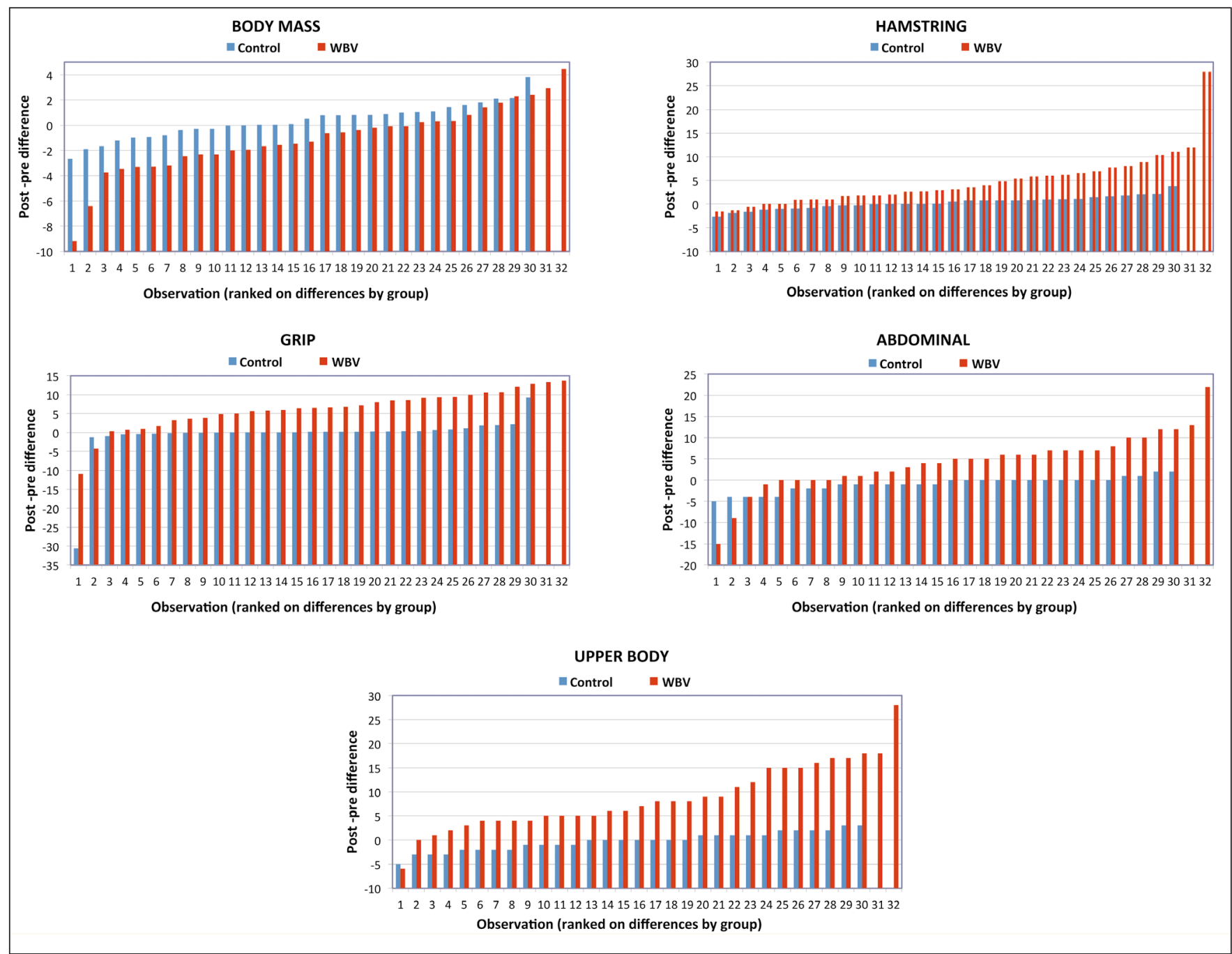

Fig. 1. Observations of all five selected dependent variables based on ranked differences by group.

\section{Upper-body muscular endurance}

Table 3 reflects that upper-body muscular endurance of the experimental group improved as the mean number of push-ups performed increased by 9 repetitions with pre- and post-test values being recorded as 24 and 33 respectively with $t=-6.88, p<0.05$ and $\chi^{2}=29.47, p<0.05$ after the 12 -week study. Conversely, the control group exhibited a decrease of 1 repetition over the entire intervention period. The mean number of push-ups for the pre-test was 20 repetitions for the control group. Posttest analysis revealed a range of $1-45$ repetitions for the control and 15 - 72 repetitions for the experimental group. Large practical significance was indicated by Cohen's $d=1.7$.

\section{Discussion}

The results indicate a significant improvement in body composition that consisted of a reduction in body mass by $2.7 \%$ in a relatively short period of 12 weeks ( $<6$ months). Evidence from several metaanalyse ${ }^{13,14}$ indicates that weight reduction programmes based purely on increasing levels of physical activity alone are not very successful (a typical loss of $0.3-1.3 \mathrm{~kg}$ over 16 weeks). The authors conclude that achieving long-term weight loss via exercise alone is rare and, together with updated recommendation for adults, ${ }^{15,16}$ propose that regular physical activity of moderate intensity for $60 \mathrm{~min} /$ day combined with caloric restriction is the most successful long-term weight management strategy. ${ }^{17}$ It should be noted that the stated optimum training time for a positive consequential effect on body composition is $60-90 \mathrm{~min} /$ day. However, this study showed that WBV training provided sufficient levels of exercise for this purpose in $30 \mathrm{~min} 3 \mathrm{x} /$ week. This decrease in overall training time shows that WBV training is effective in overcoming time constraints as a practical barrier to exercise. The long-term maintenance of weight reduction as a result of WBV training needs to be established.

The significant improvement in hamstring flexibility in the experimental group could be explained by two possible mechanisms. Firstly, the enhanced local blood flow through the muscles generated additional heat, thereby enhancing muscle elasticity and facilitating an increase in range of motion in the hamstring muscles. The second mechanism proposed is neurophysiological in nature as the vibration training elicited a tonic vibration reflex that activated the muscle spindles and led to the advancement of the stretch-reflex loop. ${ }^{18,19}$

Significant increases in upper-body muscle strength (as measured by grip strength) by using WBV training were in agreement with findings that have previously been reported for training-induced strength gains. Muscular strength scores for absolute grip strength increased by $87 \%$ over the intervention period as compared with those of their sedentary counterparts. ${ }^{20,21}$ This substantial increase in muscular strength plays an important role in health promotion, 
as it is associated with lean muscle mass and may promote better compliance with activities of daily living as laid out in the World Health Organization's global strategy on diet, physical activity and health. ${ }^{22-24}$ It could be argued that WBV training stimulated resting metabolic rate which contributes about $60-70 \%$ of daily energy expenditure and increased fat-free mass which is the main contributor to resting metabolism. Evidence shows that resistance exercises may produce a moderate increase in fat-free mass and a reduction in cardiovascular disease risk factors for some individuals independent of weight loss. ${ }^{16}$

The findings regarding abdominal and upper-body muscle endurance are in agreement with previous findings ${ }^{20,21}$ regarding an improvement in abdominal endurance after a 12-week WBV therapy programme in previously sedentary individuals. Delecluse et al. ${ }^{21}$ reported that vibratory waves irritated the primary endings of the muscle spindle that activated a larger fraction of the motor neuron pool and recruited previously inactive motor units into contraction. This resulted in a more efficient use of the force production potential of the muscle groups involved. This mechanism of motor neuron pool activation was further reinforced during WBV by the recruitment of previously inactive motor neurons, together with their activity synchronisation, and increased discharge of the neural drive, which led to greater improvements in neuromotor control during voluntary muscle contraction as evaluated in the modified sit-up and push-up assessments, respectively.

\section{Conclusion}

Although the study was conducted on a relatively small sample group over a period of 12 weeks only, it provides useful information that indicates that WBV training appears to be a safe exercise modality when structured and supervised by a health professional to enhance health-related physical fitness in sedentary adults. Thus, there is evidence to support the use of WBV training by healthcare professionals and exercise scientists as a means to increase selected health-related physical fitness variables in sedentary individuals.

Based on these results, it could be concluded that WBV training provides an effective method of exercise intervention to overcome barriers in becoming physically active and for health promotion in previously sedentary individuals. However, further research is needed to determine the long-term effects of WBV as exercise intervention for promoting health- related physical fitness in sedentary individuals, together with more direct measures of training adaptation such as heart rate and maximal oxygen uptake $\left(\mathrm{VO}_{2} \mathrm{max}\right)$.

\section{Limitations and recommendations for future research}

There is a need for further investigation into the long-term positive and negative effects of WBV therapy on the health-related components of physical fitness of anthropometrical profile, muscular flexibility, muscular strength, muscular endurance and aerobic capacity.

In addition, direct anthropometrical analyses of changes in fat mass and lean muscle mass ratios after using WBV therapy as a training modality need to be addressed.

Further research is also required in respect of the activation of core musculature during WBV therapy, since lower back pain was identified as a major debilitating factor in the wellness profile of adults during this study.
Study designs and exercise programme prescriptions should also be implemented to explore and describe the effects of WBV therapy on aerobic capacity. Pre-set heart rate requirements should be based on age-adjusted maximal heart rate values to guide the exercise programme design and prescription.

Acknowledgement. Financial assistance was received from the National Research Foundation (NRF) and the NMMU as a research grant.

\section{REFERENCES}

1. Statistics South Africa: Statistical release - P0211.2. Monthly earnings of South Africans, 2010.

2. Hoeger WK, Hoeger SA. Lifetime Physical Fitness and Wellness: A Personalised Program. 12th ed. Stanford: Wadsworth Cengage Learning, 2013.

3. Coburn JW, Malek MH. NSCA’s Essentials of Personal Training. 2nd ed. Champaign, IL: Human Kinetics, 2012.

4. Blair SN, Cheng Y, Holder JS. Is physical activity or physical fitness more important in defining health benefits? Med Sci Sports Exerc 2001;33:S379-399.

5. Physical activity guidelines and introduction. Centers for Disease Control. http://www. cdc.gov/physicalactivity/everyone/guidelines/adults.html (accessed 13 July 2011).

6. Rittweger J, Beller G, Felsenberg D. Acute physiological effects of exhaustive whole body vibration exercise in man. Clin Phys 2000;20:134-142.

7. Delecluse C, Roelants M, Diels R, Koninckx E, Verschueren S. Effects of whole body vibration training on muscle strength and sprint performance in sprint-trained athletes. Int J Sports Med 2004;26:662-668.

8. De Vos AS, Strydom H, Fouché CB, Delport CSL. Research at Grassroots for the Social Sciences and Human Service Professions. 3rd ed. Pretoria: Van Schaik, 2009: 140-208.

9. Myers J. Fundamentals of Experimental Design. 2nd ed. New York: Allyn \& Bacon, 1998:104-120.

10. Thomas JR, Nelson JK. Research Methods in Physical Activity. 3rd ed. Champaign, IL: Human Kinetics; 1996.

11. Donnely JE, Blair SN, Jackicic JM. American College of Sports Medicine position stand. Appropriate physical activity intervention strategies for weight loss and prevention of weight regain for adults. Med Sci Sports Exerc 2009;41:459-471.

12. Anderson DR, Sweeney DJ, William TA. Modern Business Statistics with Microsoft Excel. Stanford: Cengage Learning, 2003.

13. Macfarlane DJ, Thomas GN. Exercise and diet in weight management: updating what works. South African Journal of Sports Medicine 2011;23(2):57-61.

14. Bull FC, Pratt M, Shephard RJ, Lankenau B. Implementing national based action on physical activity - challenges for action and opportunities for international collaboration. Int J Health Promot Educ 2006;13(2):127-132.

15. Wing RR. Physical activity in the treatment of adulthood overweight and obesity: current evidence and research issues. Med Sci Sports Exerc 1999;31:S547-552.

16. Volek JS, Vanheest JL, Forsythe CE. Diet and exercise for weight loss: a review of current issues. Sports Med 2005;35;1-9.

17. Jakicic JM, Clark K, Coleman E. American College of Sports Medicine position stand. Appropriate intervention strategies for weight loss and prevention of weight regain for adults. Med Sci Sports Exerc 2001;33:2145-2156.

18. Carlsoo $\mathrm{S}$. The effect of vibration on the skeleton, joints and muscles: a review of literature. Appl Ergon 1982;13:251-258.

19. Eklund G, Hagbarth KE. Normal variability of the tonic vibration reflex in man. Exp Neurol 1966;16:80-92.

20. Delecluse C, Roelants M, Verschueren S. Strength increase after whole body vibration compared with resistance training. Med Sci Sport Exerc 2003;35:1033-1041.

21. Delecluse C, Roelants M, Diels R, Koninckx E, Verschueren S. Effects of whole body vibration training on muscle strength and sprint performance in sprint-trained athletes. Int J Sports Med 2006;26:662-668.

22. World Health Organization. WHO global strategy on diet, physical activity and health African regional consultation meeting report. Harare, Zimbabwe, 18-20 March 2003.

23. World Health Organization. Preventing chronic diseases: a vital investment. Geneva. Fifty-seventh World Health Assembly [WHA 57.17] held on 24 May 2004. Agenda item 12.6 for discussion on 'Global strategy on diet, physical activity and health', 2004.

24. World Health Organization. Reducing risks, promoting healthy life. The world health report. Geneva. Fifty-seventh World Health Assembly [WHA 57.17] held on 24 May 2004. Agenda item 12.6 for discussion on 'Global strategy on diet, physical activity and health', 2004. 